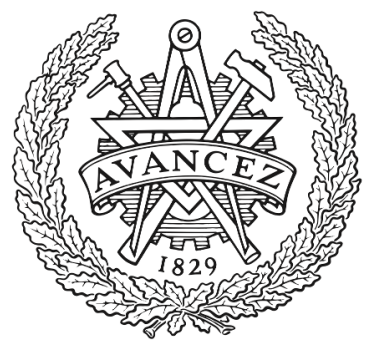

CHALMERS

UNIVERSITY OF TECHNOLOGY

\title{
Vector mode inter-modal wavelength conversion in a dispersion tailored highly nonlinear few-mode fibre
}

Downloaded from: https://research.chalmers.se, 2023-04-26 02:13 UTC

Citation for the original published paper (version of record):

Gao, J., Nazemosadat Arsanjani, S., Schröder, J. et al (2019). Vector mode inter-modal wavelength conversion in a dispersion tailored highly nonlinear few-mode fibre. IET Conference Publications, 2019(CP765). http://dx.doi.org/10.1049/cp.2019.1067

N.B. When citing this work, cite the original published paper. 


\title{
VECTOR MODE INTER-MODAL WAVELENGTH CONVERSION IN A DISPERSION TAILORED HIGHLY NONLINEAR FEW-MODE FIBRE
}

Jitao Gao ${ }^{1}$, Elham Nazemosadat ${ }^{2}$, Jochen Schröder ${ }^{2}$, Chen Yang $^{3}$, Songnian Fu ${ }^{1 *}$, Ming $^{2}$ Tang $^{1}$, Deming Liu ${ }^{1}$, Weijun Tong ${ }^{3}$, Magnus Karlsson ${ }^{2}$, Peter A Andrekson ${ }^{2}$

${ }^{1}$ National Engineering Laboratory for Next Generation Internet Access System, School of Optics and Electronic Information, Huazhong University of Science and Technology, 430074, Wuhan, China

${ }^{2}$ Photonics Laboratory, Department of Microtechnology and Nanoscience, Chalmers University of Technology, SE-41296, Gothenburg, Sweden

${ }^{3} R \& D$ Center, Yangtze Optical Fibre and Cable Joint Stock Limited Company (YOFC), 430073, Wuhan, China

*E-mail: songnian@mail.hust.edu.cn

Keywords: FEW-MODE FIBRE, NONLINEAR FIBRE OPTICS, FOUR-WAVE MIXING, HIGHLY NONLINEAR FIBRE, WAVELENGTH CONVERSION

\begin{abstract}
We present the design and fabrication of a dispersion tailored highly nonlinear few-mode fibre with an inter-modal nonlinear coefficient of $2.81(\mathrm{~W} \cdot \mathrm{km})^{-1}$, the highest reported to date. Inter-modal wavelength conversion between the $\mathrm{HE}_{21}$ and $\mathrm{TE}_{01}$ vector modes is demonstrated in the fibre.
\end{abstract}

\section{Introduction}

The nonlinear phenomenon of four-wave mixing (FWM) has been widely investigated in optical fibres, due to its applications in low-noise amplification and all-optical signal processing [1]. In recent years, with the advances in space-division multiplexing technology, inter-modal FWM processes in few mode fibres (FMFs) have also attracted much attention [25]. To date, such processes have been mostly investigated in commercial FMFs [2, 3], which have a relatively low intermodal nonlinearity. Therefore, there is interest to increase the inter-modal nonlinearity of FMFs to enhance the efficiency of such nonlinear processes. Recently, a highly nonlinear FMF, which is dispersion tailored for inter-modal FWM, has been reported [6].

In this paper, we report on the design and fabrication of a few-mode fibre (HNL-FMF), with the highest intra-modal nonlinearity reported to date, to the best of our knowledge. The HNL-FMF has a high germanium doping concentration in the core, which breaks the degeneracy between the $\mathrm{LP}_{11}$ modes and leads to different dispersion relations for the corresponding vector modes. Here, we demonstrate inter-modal wavelength conversion between two vector modes within the second mode group.

To achieve broadband FWM in single mode fibres, the pump should be placed close to the zero-dispersion wavelength and in the anomalous dispersion regime [7]. However, the phase matching condition for inter-modal FWM processes is more flexible, due to the different dispersion properties of different modes. The phase mismatch in these processes is given by [4]

$$
\Delta \beta \approx\left(\omega_{s}-\omega_{p 1}\right)\left[\beta_{1}^{a}\left(\frac{\omega_{s}+\omega_{p 1}}{2}\right)-\beta_{1}^{b}\left(\frac{\omega_{i}+\omega_{p 2}}{2}\right)\right],
$$

where $\beta_{1}^{m}(\omega)$ represents the inverse group velocity of mode $m$ at angular frequency $\omega$, while $p 1, p 2, s$ and $i$ represent the interacting pumps, signal and the generated idler, respectively. Thus, to have phase matching, $\beta_{1}$ at the average frequencies of the two waves in each spatial mode should be equal. Bragg scattering (BS) and phase conjugation (PC) are the two types of inter-modal FWM processes that can result in wavelength and mode conversion. When the interacting modes have similar chromatic dispersion properties, BS has a relatively wider phase matching bandwidth compared to PC [3]. Along with the inter-modal process, modulational instability (MI) which is an intra-modal FWM process could also happen if the interacting waves are phase matched, as shown in Fig 1(a).

\section{Fibre Design and Characterisation}

We designed and fabricated a dispersion tailored highly nonlinear few-mode fibre which supports two mode groups, with a graded index circular core, surrounded by a trench and the cladding [8]. The optimised refractive index profile design is shown in Fig 1(b), as well as the measured refractive index profile of the fabricated HNL-FMF. The core with a radius of $4.3 \mu \mathrm{m}$ is highly doped with germanium ( $25 \mathrm{~mol} . \%$ at the centre of the core), to obtain high nonlinearity. Meanwhile, the relatively large refractive index difference between the core and cladding breaks the degeneracy in the second mode group, resulting in different propagation constants of $\mathrm{HE}_{21}, \mathrm{TE}_{01}$ and $\mathrm{TM}_{01}$ modes, hence providing the possibility of obtaining inter-modal FWM between the vector modes within one mode group. Although the vector modes in the second mode group share almost the same spatial distribution, the polarisation distributions and measured relative inverse group velocities $\left(\Delta \beta_{1}\right)$ 


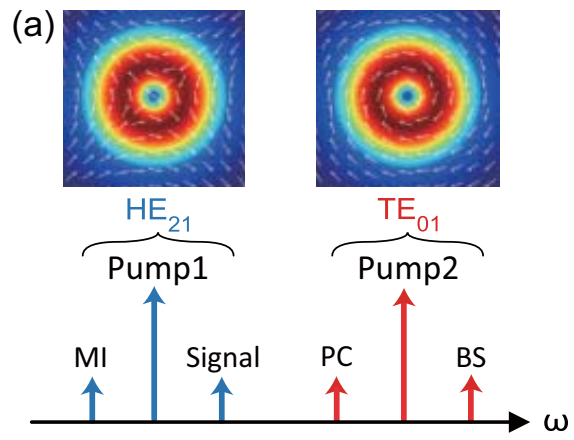

(b)

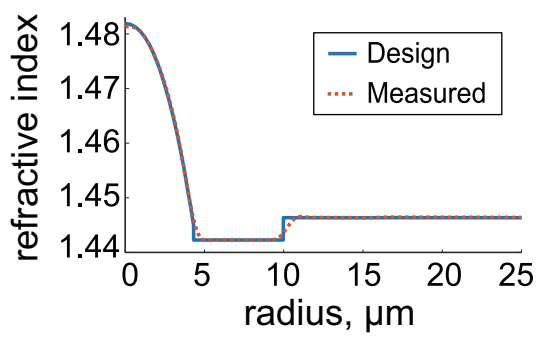

(c)

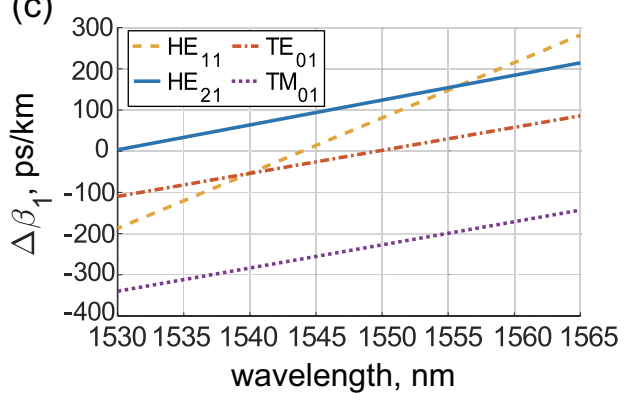

Fig. 1 (a) Schematic of the intra- and inter-modal FWM processes occurring when Pump1 and Signal in mode $\mathrm{HE}_{21}$ and Pump2 in mode $\mathrm{TE}_{01}$ are launched in to the HNL-FMF. The intra- and inter-modal generated idlers are $\mathrm{MI}$ in $\mathrm{HE}_{21}$ mode, and $\mathrm{BS}$ and $\mathrm{PC}$ in $\mathrm{TE}_{01}$ mode, respectively. The spatial and polarisation distributions of the interacting modes are also shown. (b) Designed and measured refractive index profiles of the HNL-FMF. (c) Measured relative inverse group velocities versus wavelength of all the guided vector modes.

are different, as shown in Fig 1(a) and (c). It is worth noting that $\Delta \beta_{1}$ curves of the three vector modes in the second mode group are nearly parallel, indicating similar group velocity dispersion $\beta_{2}\left(-7.18,-7.17\right.$ and $-7.17 \mathrm{ps}^{2} / \mathrm{km}$ at $1550 \mathrm{~nm}$ for $\mathrm{HE}_{21}, \mathrm{TE}_{01}$ and $\mathrm{TM}_{01}$ modes, respectively). Consequently, their dispersion properties makes them suitable for broadband BS process [3].

The effective areas of the first and second mode groups are 21.3 and $42.9 \mu \mathrm{m}^{2}$ respectively, while the intra-modal nonlinear coefficients $\gamma$ of them are 5.68 and $2.81(\mathrm{~W} \cdot \mathrm{km})^{-1}$ respectively, measured with the method described in [9]. Since the inter-modal nonlinear coefficient is related to the overlap integral of the optical field distribution of the interacting modes, the inter-modal nonlinear coefficient between any two of the vector modes is approximately equal to the intra-modal nonlinear coefficient of the second mode group. Thus, intermodal nonlinear coefficient is also $2.81(\mathrm{~W} \cdot \mathrm{km})^{-1}$. The attenuation coefficients are measured to be $0.59 \mathrm{~dB} / \mathrm{km}$ and $0.65 \mathrm{~dB} / \mathrm{km}$ for the first and second mode group, respectively.

\section{Experimental Setup}

The experimental setup of our vector-mode inter-modal FWM is shown in Fig 2. Pump1 and Signal launched into $\mathrm{HE}_{21}$ mode are set to be at $1543 \mathrm{~nm}$ and $1543.25 \mathrm{~nm}$, respectively. Pump2 is launched into $\mathrm{TE}_{01}$ mode, and to achieve phase matching its wavelength is set to $1565 \mathrm{~nm}$ (see Fig 1(c)). For Pump1 and Signal, light coming out of the continuous-wave (CW) lasers are adjusted to be co-polarised by the polarisation controllers (PCs) right after the lasers, then coupled together into an erbium-doped fibre amplifier (EDFA) by a 90:10 polarisation maintaining coupler. A tunable optical band-pass filter (OBPF) is used after the EDFA in order to filter out the outband amplified spontaneous emission noise. We use two spatial light modulators (SLMs) based on liquid crystal on silicon (LCoS) as the mode multiplexer (MMUX) and demultiplexer (MDMUX), to realise mode selective excitation using optimised phase masks. The interacting waves propagate through the 2-km HNL-FMF, where BS and PC idlers are generated in the $\mathrm{TE}_{01}$ mode. It should be noted that mode coupling occurs between the vector modes in the second mode group, so the power in the pumps and signal are a mix of these modes. However, only the beam powers in the phase matched vector modes will be involved in the inter-modal FWM. By changing the phase mask in the MDMUX, we can observe the output spectra of different modes with an optical spectrum analyser (OSA).

The power of each pump after the EDFA is set to be 28.2 $\mathrm{dBm}$ and the Signal power is $12 \mathrm{~dB}$ lower. Meanwhile, the MMUX together with the OBPF and all the fibre connectors has a total loss of $12.2 \mathrm{dBm}$ for each input port when exciting $\mathrm{HE}_{21}$ or $\mathrm{TE}_{01}$ mode. Thus, the actual pump power launched into each mode is $16 \mathrm{dBm}$.

\section{Experimental Results and Discussion}

By using different phase masks at the MDMUX, optimised for different vector modes, the output spectra after 2-km HNLFMF transmission are shown in Fig 3. In Fig 3(a), for the waves

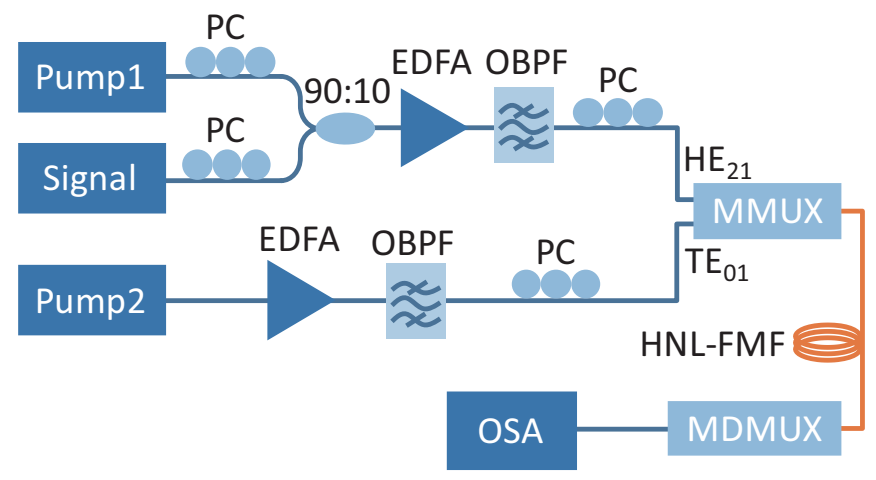

Fig. 2 Experimental setup of vector mode inter-modal wavelength conversion. Blue and orange lines correspond to single mode and few-mode fibre links, respectively. 


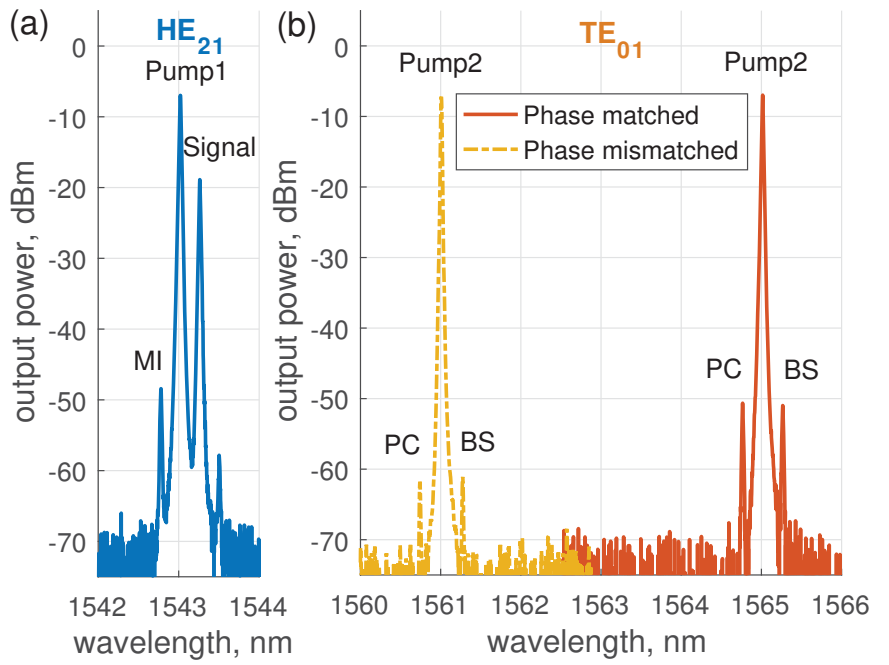

Fig. 3 Output spectra of $\mathrm{HE}_{21}$ and $\mathrm{TE}_{01}$ modes after mode demultiplexing, observed in different wavelength windows (a) and (b), respectively. Solid curves represent phase matched inter-modal FWM, while the dashed curve in (b) represents the inter-modal FWM with a phase mismatch.

in $\mathrm{HE}_{21}$ mode, we can see an intra-modal idler generated by $\mathrm{MI}$ process with a conversion efficiency (CE, defined as the power ratio between the output idler and output Signal) of -29.9 dB. While in Fig 3(b) around $1565 \mathrm{~nm}$, the BS and PC idlers are generated $0.25 \mathrm{~nm}$ away from Pump2, with CEs of $-32.1 \mathrm{~dB}$ and $-31.8 \mathrm{~dB}$, respectively. The similar CEs of BS, PC and MI processes when Signal is set close to Pump1 indicate similar nonlinear coefficients for intra- and inter-modal interactions.

Given that the vector modes are within one mode group and have mode coupling between them, one might think the process captured here is a dual-pump intra-modal FWM interaction not inter-modal FWM. To demonstrate that it is indeed an intermodal process, we moved Pump2 closer to Pump1, to $1561 \mathrm{~nm}$, which is far from the phase matching wavelength of the intermodal FWM processes. In the case of a dual-pump intra-modal FWM process, the FWM efficiency should have increased, since the detuning between the two pumps is smaller and all the interacting modes are in the anomalous dispersion regime $\left(\beta_{2}\right.$ $<0)$ and far from zero-dispersion wavelength [10]. However, as observed in Fig 3(b), the idler powers in this case are significantly lower than the ones when Pump2 is at $1565 \mathrm{~nm}$, proving that the process is inter-modal FWM. Thus, a wavelength and vector mode conversion is accomplished by inter-modal FWM between vector modes. If we make use of the $\mathrm{TM}_{01}$ mode, a wider-band wavelength conversion over $\mathrm{C}+\mathrm{L}$ band would be possible.

Fig 4 shows how CEs of BS and PC processes change when sweeping the Signal and changing the Signal to Pump1 wavelength detuning. It is obvious that BS has a broader $\mathrm{CE}$ bandwidth compared to the PC process. Taking a 6-dB bandwidth into consideration, the bandwidths of $\mathrm{BS}$ and $\mathrm{PC}$

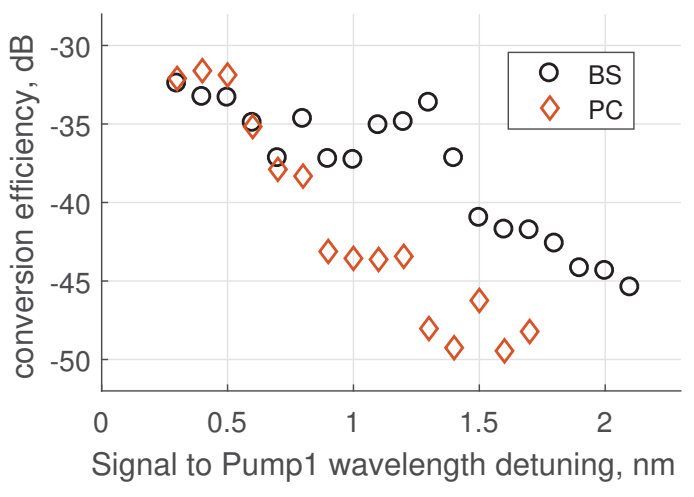

Fig. 4 CEs of the BS and PC processes, measured under different Signal to Pump1 wavelength detunings varying from 0.3 to $2.1 \mathrm{~nm}$ with a wavelength interval of $0.1 \mathrm{~nm}$.

processes are $1.4 \mathrm{~nm}$ and $0.8 \mathrm{~nm}$ respectively, due to the similar chromatic dispersion properties of the interacting vector modes. In addition, there is a trade-off between the fibre length and the bandwidth of the inter-modal processes [4]. Due to the loss induced by the SLM in our system, a relatively long fibre length was needed to observe the inter-modal nonlinearities. However, it is possible to shorten the fibre and increase the bandwidth of the inter-modal FWM processes by increasing the pump powers or using other MMUX/MDMUX schemes with smaller losses.

\section{Conclusion}

An inter-modal vector mode wavelength conversion with a wavelength separation of $22 \mathrm{~nm}$ is accomplished between $\mathrm{HE}_{21}$ and $\mathrm{TE}_{01}$ modes, by implementing inter-modal FWM in a newly designed and fabricated HNL-FMF. We provide a novel solution to vector mode generation using inter-modal FWM. The fibre also shows great potential in all-optical signal processing, such as vector mode conversion, mode exchanging and wavelength multicasting.

\section{Acknowledgements}

This work was supported in part by the National Natural Science Foundation of China (NSFC) under Grant 61711530043, in part by the Swedish Research Council (VR) under Grant Agreement 2015-00535 and in part by the Swedish foundation for international cooperation in higher education (STINT) via contract CH2016-6754. 


\section{References}

[1] Tong, Z., Radic, S.: 'Low-noise optical amplification and signal processing in parametric devices', Adv. Opt. Photon., 2013, 5, (3), pp. 318-384

[2] Essiambre, R.J., Mestre, M.A., Ryf, R., et al.: 'Experimental investigation of inter-modal four-wave mixing in few-mode fibers', IEEE Photon. Technol. Lett., 2013, 25, (6), pp. 539-542

[3] Friis, S.M.M., Begleris, I., Jung, Y., et al.: 'Inter-modal four-wave mixing study in a two-mode fiber', Opt. Express, 2016, 24, (26), pp. 30338-30349

[4] Xiao, Y., Essiambre, R.J., Desgroseilliers, M., et al.: 'Theory of intermodal four-wave mixing with random linear mode coupling in few-mode fibers', Opt. Express, 2014, 22, (26), pp. 32039-32059

[5] Nazemosadat, E., Pourbeyram, H., Mafi, A.: 'Phase matching for spontaneous frequency conversion via fourwave mixing in graded-index multimode optical fibers', J. Opt. Soc. Am. B, 2016, 33, (2), pp. 144-150

[6] Rademacher, G., Luís, R.S., Puttnam, B.J., et al.: 'Wideband intermodal wavelength conversion in a dispersion engineered highly nonlinear fmf'. In: Optical Fiber Communication Conference (OFC) 2019. (Optical Society of America, 2019). p. W1C.4

[7] Agrawal, G.P.: 'Nonlinear fiber optics, 4th ed.'. (San Diego: Academic Press, 2007).

[8] Nazemosadat, E., Lorences.Riesgo, A., Karlsson, M., et al.: 'Design of highly nonlinear few-mode fiber for c-band optical parametric amplification', J. Lightw. Technol., 2017, 35, (14), pp. 2810-2817

[9] Boskovic, A., Chernikov, S.V., Taylor, J.R., et al.: 'Direct continuous-wave measurement of $\mathrm{n} 2$ in various types of telecommunication fiber at $1.55 \mu \mathrm{m}$ ', Opt. Lett., 1996, 21, (24), pp. 1966-1968

[10] Marhic, M.E., Park, Y., Yang, F.S., et al.: 'Widely tunable spectrum translation and wavelength exchange by fourwave mixing in optical fibers', Opt. Lett., 1996, 21, (23), pp. 1906-1908 